\title{
Wetting layer of copper on the tantalum (001) surface
}

\author{
Maxime Dupraz,,${ }^{1, *}$ Roberta Poloni, ${ }^{1}$ Kitti Ratter,${ }^{1,2}$ David Rodney,${ }^{1,3}$ Maurizio De Santis, ${ }^{2}$ Bruno Gilles, ${ }^{1}$ \\ Guillaume Beutier, ${ }^{1, \dagger}$ and Marc Verdier ${ }^{1}$ \\ ${ }^{1}$ Univ. Grenoble Alpes, CNRS, SIMAP, F-38000 Grenoble, France \\ ${ }^{2}$ CNRS, Inst NEEL, F-38000 Grenoble, France \\ ${ }^{3}$ Institut Lumière Matière, Université Lyon 1, CNRS, UMR 5306, F-69622 Villerbanne, France \\ (Received 17 June 2016; revised manuscript received 6 November 2016; published 22 December 2016)
}

\begin{abstract}
The heteroepitaxial interface formed by copper deposited onto the tantalum (001) surface is studied by surface $\mathrm{x}$-ray diffraction and $a b$ initio calculations. The analysis of the crystal truncation rods reveals the presence of a wetting layer of copper made of two atomic planes pseudomorphic to the tantalum substrate, with the upper most atomic planes significantly deformed. These findings are in total agreement with the results of density-functional-theory calculations. The presence of the wetting layer confirms a Stranski-Krastanov growth mode and is thought to explain the extremely fast atomic diffusion of copper during the dewetting process in the solid state at high temperature.
\end{abstract}

DOI: 10.1103/PhysRevB.94.235427

\section{INTRODUCTION}

Copper is nowadays a widely used interconnected metal in integrated circuits devices. However, the rapid diffusion of copper through silicon and its poor adhesion to silicon oxide led to the investigation of reliable diffusion barriers to isolate the copper interconnects from the silicon chips. Among the diffusion barrier metals, tantalum is one of the most interesting candidates and the past two decades have seen an increasing interest for the study of the $\mathrm{Cu}$-Ta system [1-4]. On top of its technological interest, the $\mathrm{Cu}$-Ta system is also a prime example of a strongly heterogeneous film-substrate system with almost zero mutual solubility [5], different crystal structure [face-centered cubic (fcc) $\mathrm{Cu}$ vs. body-centered cubic (bcc) Ta], and very different lattice parameters. An atomic scale understanding of the growth mode, structure, and thermodynamic stability of $\mathrm{Cu}$ thin films on Ta substrates is thus of both fundamental and technological interest.

The $\mathrm{Cu}-\mathrm{Ta}$ interface is a good example of a bimetallic system with a Stranski-Krastanov growth mode [6] and can be described as a growth of three-dimensional (3D) islands on top of a thin wetting layer. The structure and morphology of the $\mathrm{Cu}$-Ta interface is, however, difficult to predict, and the study of the stability and wetting behavior of thin $\mathrm{Cu}$ films deposited on a Ta substrate has been the subject of several experimental investigations and controversial observations over the past few years. All studies agree that $\mathrm{Cu}$ thin films are metastable on Ta and tend to dewet into 3D structures, leaving a thin wetting layer: This general behavior has been observed on polycrystalline bcc Ta [7] and on single-crystal surfaces of the bcc [8] and $\beta$ structures [9].

Most studies deal with the (110) surface of bcc tantalum, which is the dense plane of the stable structure. It has been established that ultrathin $\mathrm{Cu}$ films form a stable pseudomorphic (PM) layer when deposited on top of a Ta (110) substrate for a coverage up to 1.22 monolayers (ML) [10].

\footnotetext{
*Current address: Swiss Light Source, Paul Scherrer Institut, CH5232, Villigen PSI, Switzerland

${ }^{\dagger}$ guillaume.beutier@simap.grenoble-inp.fr
}

Additional $\mathrm{Cu}$ layers are unstable and dewet in the solid state at temperatures and with kinetics depending strongly on contamination [7,9] and on the film thickness [8]. On a clean Ta surface in an ultra high vacuum (UHV) environment a $\mathrm{Cu}$ monolayer is stable up to $1000 \mathrm{~K}$, the temperature at which diffusion into the polycrystalline bulk occurs [7]. It was shown experimentally [9] and with ab initio calculations [11] that when $\mathrm{Cu}$ films dewet from $\mathrm{Ta}(110)$ substrates, they leave a single stable monolayer on top of which 3D Cu islands form. The crystallographic orientation of the dewetted copper on $\mathrm{Ta}(110)$ follows the Nishiyama-Wassermann relationship: $\mathrm{Ta}(110)[110] / / \mathrm{Cu}(111)[112]$ [8].

The growth mode and wetting behavior of $\mathrm{Cu}$ thin films on $\mathrm{Ta}(001)$ are not well documented. The main experimental UHV investigation, performed by Venugopal et al., reported the dewetting of $\mathrm{Cu}$ thin films deposited on $\mathrm{Ta}(001)$ [8]. They observed, by x-ray diffraction, both the (111) and (001) out-of-plane orientations of the $\mathrm{Cu}$ islands and found, based on the morphologies observed by scanning electron microscopy (SEM), that the latter is largely predominant. On the other hand, Francis et al. observed a strong (111) texture for copper thin films sputtered on Ta(001) [12]. We also reported the dewetting of highly strained $\mathrm{Cu}$ islands with the $\mathrm{Cu}(001)[110] / / \mathrm{Ta}(001)[100]$ orientation relationship [13]. Venugopal et al. evidenced a remaining wetting layer between the dewetted islands, with a thickness estimated to less than $5 \AA$, corresponding to one or two ML [8].

There is, to our knowledge, no experimental determination of its structure to date, but several numerical works have addressed the question. A first study employing molecular dynamics (MD) predicted that the $\mathrm{Cu}(111)-\mathrm{Ta}(001)$ interface is more stable than $\mathrm{Cu}(001)-\mathrm{Ta}(001)$ and $\mathrm{Cu}(110)-\mathrm{Ta}(001)$ interfaces [14]. More relevant to the dewetting of a single $\mathrm{Cu}$ thin film, Lazic et al. performed MD calculations of the deposition of $\mathrm{Cu}$ on a $\mathrm{Ta}(001)$ surface and found an fcc structure with (111) texture from the third $\mathrm{Cu}$ ML, on top of a PM ML and a complex intermediate ML [15]. Only Hashibon et al. [16] developed cross potentials for $\mathrm{Cu}-\mathrm{Ta}$ from $a b$ initio calculations to describe the dewettting of $\mathrm{Cu}$ on the $\mathrm{Ta}(110)$ surface. There is thus so far no numerical work predicting the $\mathrm{Cu}(001)[110] / / \mathrm{Ta}(001)[100]$ orientation 
relationship evidenced by Venugopal et al. and Beutier et al. $[8,13]$. We note that in the works mentioned above, $a b$ initio calculations using the density functional theory (DFT) were not directly employed to assess the stability of various interfaces but used indirectly to build interatomic potentials for MD simulations.

In this study, we used surface x-ray diffraction (SXRD) and DFT calculations to address the presence and structure of a wetting layer of $\mathrm{Cu}$ on the $\mathrm{Ta}(001)$ surface. We measured SXRD in situ while depositing and dewetting a thin $\mathrm{Cu}$ layer on a $\mathrm{Ta}(001)$ surface. The measurements show unambiguously the presence of two PM Cu layers. The interplanar spacing between the $\mathrm{Cu}$ layers and the uppermost Ta layers is also determined. DFT calculations in the local density approximation were used to calculate the excess interface energy of a variety of configurations with different numbers of $\mathrm{Cu} M \mathrm{ML}$ and different structures. A very good agreement is found between both approaches.

\section{SURFACE X-RAY DIFFRACTION}

SXRD is a powerful probe of the structure of crystalline surfaces [17]. Sharp interfaces give rise to crystal truncation rods (CTR) in reciprocal space [18] that are particularly useful to evidence PM wetting layers [19]. Indeed, the scattering amplitude from PM layers is enhanced by interference with the scattering amplitude from the substrate in the vicinity of the in-plane Bragg reflections of the latter, allowing a quantitative determination of the number of atomic layers and their interplanar spacing.

The experiment was carried out at beamline BM32 of the European Synchrotron Radiation Facility (ESRF) on the SUV end station [20]. The latter consists of a UHV chamber fully equipped for sample preparation, mounted on a $z$-axis diffractometer [21]. Further degrees of freedom are available to align the sample. While the deposition of copper by molecular beam epitaxy (MBE) and the dewetting could be performed in situ, the preparation of the $\mathrm{Ta}(001)$ surface requires flash annealing at temperatures above $2000 \mathrm{~K}$, which cannot be done with the setup of the beamline. The surface preparation was thus performed in the UHV chamber of the SIMaP laboratory and then transferred to the beamline chamber.

A high purity (more than 99.9999\%) Ta crystal, cut and polished to less than $0.1^{\circ}$ along the (001) plane, was purchased from MaTecK $\mathrm{GmbH}$. The surface was prepared by repeated cycles of argon sputtering and flash annealing above $2000 \mathrm{~K}$ to remove oxygen and carbon contamination [22]. The atomic flatness of the surface was monitored by low-energy electron diffraction, and the minimum level of oxygen or carbon contamination was checked by x-ray photoelectron spectrometry. A protective 5-nm copper layer was then deposited to cover the

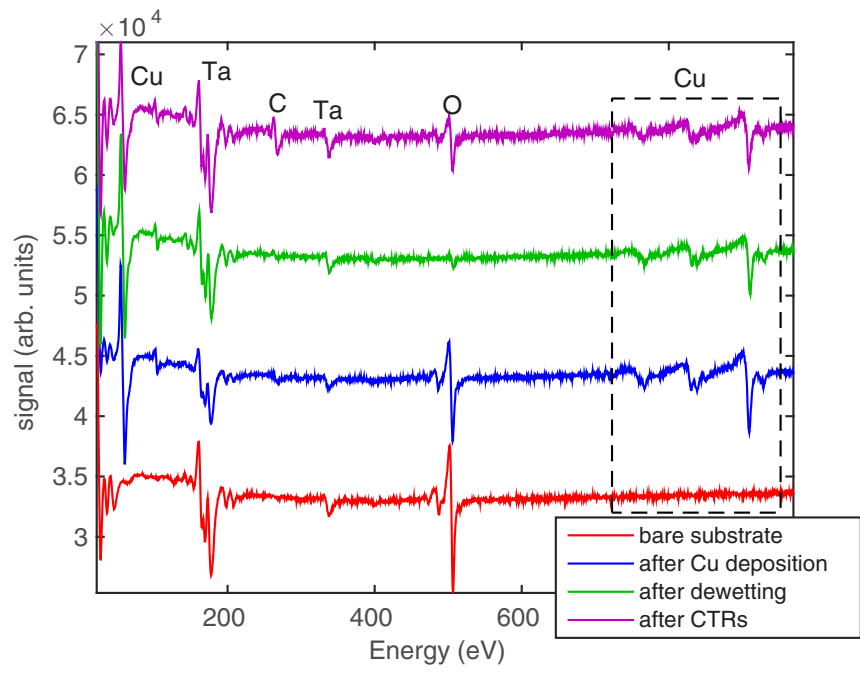

FIG. 1. Auger spectra at various stages of the experiment (here data set D).

Ta surface during the transfer from the laboratory chamber to the UHV beamline chamber, where it was removed by evaporation above $1300 \mathrm{~K}$. The transfer was done mostly in primary vacuum, with a short exposure (a few seconds) to air. We were thus able to obtain a clean Ta bare substrate surface (Fig. 1).

Four data sets (A, B, C, and D) were collected during two experimental sessions. The conditions of preparation of the Ta surface and the $\mathrm{Cu}$ deposition and dewetting differed for the four data sets. The relevant parameters are summarized in Table I. The same substrate was used for the $(A, B, C)$ data set according to the procedure detailed above. For data set $\mathrm{D}$, the substrate from experiment $\mathrm{C}$ was reused and thus did not undergo a high-temperature flash annealing preparation. The remaining copper was evaporated in situ until a clean surface was obtained ( $3 \mathrm{~min}$ at $1370 \mathrm{~K}$ ). A characteristic signature of Oxygen can be seen in the Auger spectrum of the bare Ta substrate, at $510 \mathrm{eV}$. It corresponds to about a third of an atomic layer. These are adsorbed oxygen atoms, responsible for a surface reconstruction [23], that desorb during the dewetting (green line in Fig. 1).

Starting from the bare substrate at room temperature, a few (exact numbers depending on data sets) ML of copper were deposited in situ at low rate $(\sim 5 \mathrm{~min} / \mathrm{ML})$. For data set $\mathrm{A}$ only, the $\mathrm{Cu}$ thin film was then sputtered by argon for $20 \mathrm{~min}$. The dewetting was then performed in situ at high temperature (1020-1070 K), using the furnace of the sample stage. The temperature was monitored with a pyrometer and the base pressure into the chamber was carefully checked to avoid copper evaporation from the surface. The dewetting was monitored by the rocking curve of the $\mathrm{Cu} 111$ reflections: The

TABLE I. Sample preparation parameters for the four data sets.

\begin{tabular}{lccccc}
\hline \hline Data set & Session & Substrate restauration & Cu thickness & Argon sputtering & Dewetting and maturation \\
\hline A & April 2014 & Yes & $\sim 9 \mathrm{~nm}$ & Yes & $20 \mathrm{~min}$. at $1020 \mathrm{~K}$ \\
B & Sept 2014 & Yes & $22 \mathrm{ML}(\sim 4 \mathrm{~nm})$ & No & 5 min. at $1070 \mathrm{~K}+1 \mathrm{~min}$. at $1100 \mathrm{~K}$ \\
C & Sept 2014 & Yes & $22 \mathrm{ML}(\sim 4 \mathrm{~nm})$ & No & 5 min. at $1070 \mathrm{~K}+1 \mathrm{~min}$. at $1100 \mathrm{~K}$ \\
D & Sept 2014 & No & $3 \mathrm{ML}$ & No & 1 min. at $1050 \mathrm{~K}$ \\
\hline \hline
\end{tabular}


TABLE II. Contamination of the surface before and after the CTR measurements. The age of the sample is counted from the end of the dewetting.

\begin{tabular}{|c|c|c|c|c|c|c|c|c|}
\hline \multirow[b]{2}{*}{ Data set } & \multicolumn{2}{|c|}{ Oxygen contamination } & \multicolumn{2}{|c|}{ Carbon contamination } & \multirow[b]{2}{*}{ Age at start of $11 L \mathrm{CTR}$} & \multirow[b]{2}{*}{$\Delta(11 L)$} & \multirow[b]{2}{*}{ Age at start of $10 L \mathrm{CTR}$} & \multirow[b]{2}{*}{$\Delta(10 L)$} \\
\hline & Before CTR & After CTR & Before CTR & After CTR & & & & \\
\hline A & Very low & Medium & No & Strong & $3.5 \mathrm{~h}$ & 0.073 & $0.5 \mathrm{~h}$ & 0.116 \\
\hline B & $\mathrm{L}$ & Medium & No & Low & $2 \mathrm{~h}$ & 0.103 & $3.5 \mathrm{~h}$ & 0.099 \\
\hline $\mathrm{C}$ & Low & Medium & No & Low & $0.5 \mathrm{~h}$ & 0.089 & $3.5 \mathrm{~h}$ & 0.079 \\
\hline $\mathrm{D}$ & Low & Medium & No & Medium & $0.5 \mathrm{~h}$ & 0.078 & $3.5 \mathrm{~h}$ & 0.083 \\
\hline
\end{tabular}

beginning of dewetting into islands yield a sharp increase of the peak intensity and a decrease of the rocking curve width. After dewetting, the Auger spectra show an increase of the ratio between low-energy electrons and high-energy electrons (Fig. 1). This can be explained by the presence of a thin wetting layer between the islands. The $\mathrm{Cu}(920 \mathrm{eV})-\mathrm{Cu}(60$ $\mathrm{eV}$ ) intensity ratio does not increase despite the dewetting at high temperature, which suggests that diffusion of copper into the substrate can be excluded [7]. At this stage, the oxygen signature has disappeared, due to the desorption of the adatoms on heating [23]. No other contamination is evidenced yet.

The Auger spectra were remeasured after the CTRs measurements, evidencing oxygen and carbon contaminations of more or less importance depending on the data set (Fig. 1). The level of contamination is summarized in Table II. Because of the slow contamination, the delay between the dewetting and the CTR measurements could be an important parameter. This issue can be addressed by comparing the four data sets.

The $\mathrm{x}$-ray source is a bending magnet and the monochromator is a $\mathrm{Si}(111)$ double crystal, with the second crystal bent to give sagittal focusing on the sample. The vertical focusing is provided by a mirror, resulting in a spot size of about $0.4 \times 0.3 \mathrm{~mm}^{2}$. The measurements were performed at photon energy of $22 \mathrm{keV}$. The detector was a Maxipix camera [24]. In order to reduce the thermal diffuse scattering from the bulk, the penetration of $\mathrm{x}$ rays into the sample has to be kept as small as possible. This can be achieved by using a low glancing angle $\alpha$ of the incoming X-ray beam. In this experiment $\alpha$ was set to $0.6^{\circ}$, a value about 3 times the critical angle of total external reflection $\left(0.194^{\circ}\right.$ for Ta at $\left.22 \mathrm{keV}\right)$.

The atomic structure of the surface can be determined from the structure factors measured along the CTRs, extracted from the integrated intensities measured by scanning across the CTRs with the angle $\omega[20,25]$ over a range of $\pm 5^{\circ}$. This relatively large range ensures an accurate measurement of the background level. The CTRs are thereafter indexed in the reciprocal lattice units (rlu) of bcc tantalum, with a (001) surface. The $10 L$ and $11 L$ CTRs $(0<L<3)$ were measured for each data set. All measurements were averaged over two equivalent CTRs, based on the overall tetragonal symmetry of the surface. The agreement factor $\Delta$ (average-normalized variance) between equivalent CTRs is reported in Table II. The error bars shown in the figures below are calculated as a mix of the variance between equivalent reflections and of the statistical error [26].

For all data sets, clear interferences can be seen on the CTRs, revealing the presence of epitaxial layers (Fig. 2). A straitforward conclusion is the absence of amorphization of copper, such as observed by Kwon et al. at the interface with $\beta$ - tantalum on annealing [27]. Consistently with our result, they do not see such amorphization but an atomically sharp interface with bcc tantalum, of which the crystallographic orientation is not given.

Auger spectroscopy revealed a strong carbon contamination in data set A after the CTR measurements. This is clearly seen on both CTRs from this data set, which show less contrasted features than the other three data sets and a shift of the minimum of the $11 \mathrm{~L} \mathrm{CTR} \mathrm{(Fig.} \mathrm{2).} \mathrm{The} \mathrm{impact} \mathrm{of} \mathrm{carbon}$
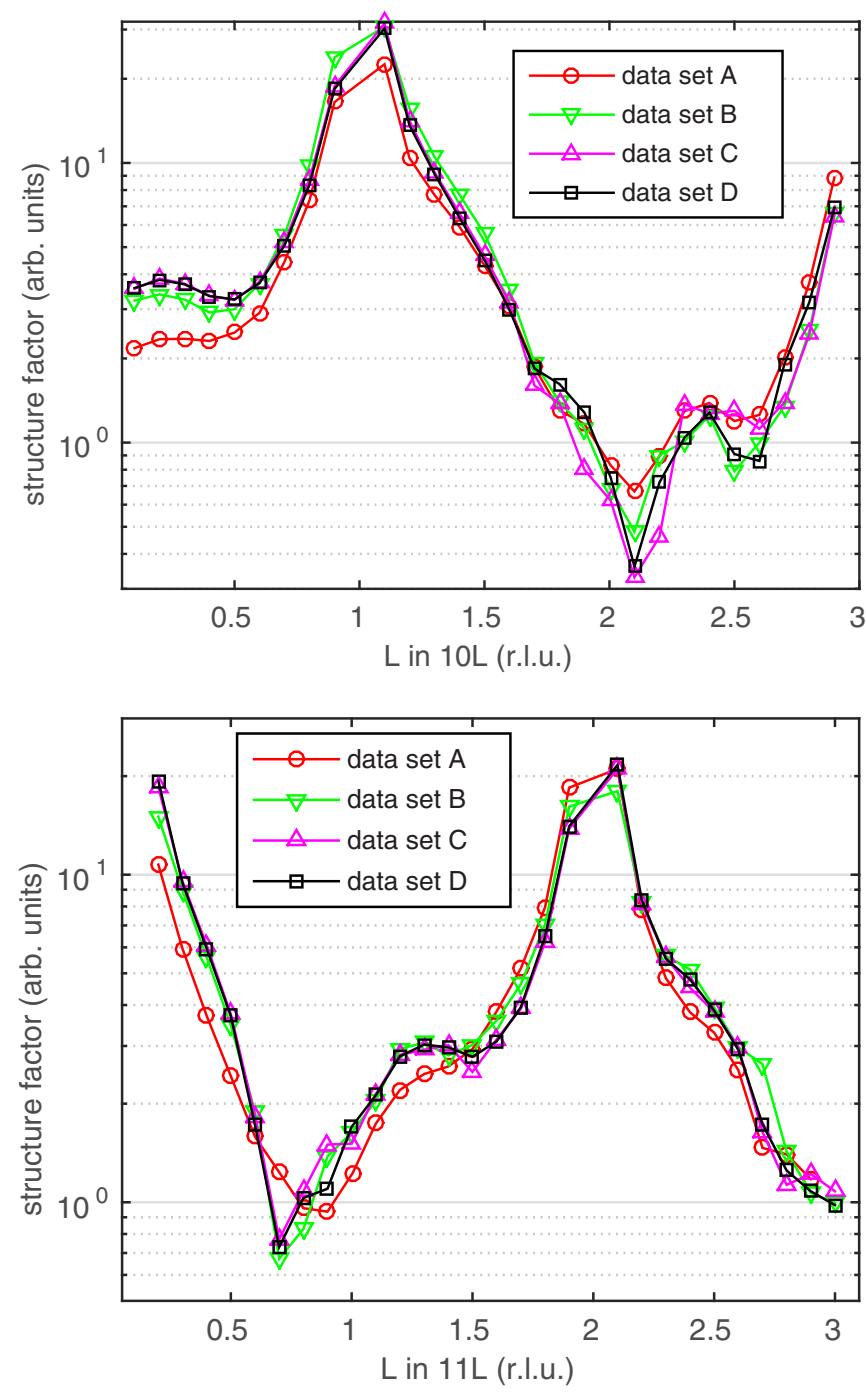

FIG. 2. $10 \mathrm{~L}$ and $11 \mathrm{~L}$ CTRs for four different data sets with different states of surface contamination. Error bars have been omitted to facilitate the visualization. 
contamination seems to be lower on the $10 L$ CTR, which was measured shortly after dewetting, while the $11 L$ was measured $3 \mathrm{~h}$ later. Such contamination could decrease the coherent fraction of the wetting layer. The other three data sets show very similar CTRs. The effect of oxygen contamination is difficult to evaluate, because the contamination level is similar for the four data sets.

After the last SXRD measurement, the surface of the sample has been imaged by optical and atomic force microscopy and confirm the dewetting of the excess copper into 3D islands similar to those observed in Refs. $[8,13]$. Their density is very low, in agreement with the small quantity of copper deposited for this data set (estimated to three ML, among which only one dewetted), with an average $30-\mu \mathrm{m}$ distance between islands.

\section{DENSITY-FUNCTIONAL-THEORY CALCULATIONS}

From the measured CTRs, the precise determination of the structure of the interface is in principle obtained by the fitting of the experimental data. DFT calculations were performed to gain further insight in the experimental results. The agreement between the interfaces predicted from the experimental data and the DFT calculations is quantified and discussed in detail.

Total energy calculations were performed using the localdensity approximation to the exchange and correlation and norm-conserving Martins-Troullier pseudo potentials [28] as implemented in the PWSCF code [29]. The wave function and charge density cutoffs were taken as $90 \mathrm{Ry}$ and $360 \mathrm{Ry}$, respectively. Atomic relaxation was performed until atomic forces were less than $0.03 \mathrm{eV} / \AA$. The bulk structures of bcc Ta and fcc $\mathrm{Cu}$ were fully optimized using a $12 \times 12 \times 12$ MonkhorstPack grid [30] for the integration of the irreducible Brillouin zone leading to $a_{\mathrm{Cu}}=3.554 \AA$ and $a_{\mathrm{Ta}}=3.235 \AA$. These values are about $2 \%$ smaller than the experimental values $(3.615 \AA$ and $3.301 \AA$ respectively at room temperature), consistent with the local density approximation. Importantly, the ratio of calculated lattice parameters is $\left(a_{\mathrm{Cu}}-a_{\mathrm{Ta}}\right) / a_{\mathrm{Ta}}=9.9 \%$, in good agreement with the experimental value $(9.5 \%)$. The slab calculations of a few PM Cu layers described in the following section employed a $12 \times 12 \times 1$ Monkhorst-Pack grid.

In order to determine the stability and structure of a $\mathrm{Cu}$ thin film on $\mathrm{Ta}(001)$, interfaces containing one to six PM layers were modeled using a periodic slab configuration (Fig. 3). It consists of a stack of $14 \mathrm{Ta}(001)$ planes covered by one to six bcc $\mathrm{Cu}$ layers on both sides, leading to two equivalent $\mathrm{Cu}-\mathrm{Ta}$ interfaces. Each layer was modeled using only one atom per unit cell by taking advantage of the in-plane periodic boundary conditions. A thick vacuum region of about $15 \AA$ was added along $z$ between the two $\mathrm{Cu}$ films in order to ensure a negligible interaction between the two free surfaces. The structural relaxation was computed by relaxing the $z$ coordinate of all atoms of the supercell.

The thermodynamical stability of $\mathrm{Cu}$ thin films was evaluated by computing the excess interface energy as the energy difference between the fully relaxed configuration for each model and the configuration with the same number of atoms in their bulk environments. It can thus be written as follows:

$$
\gamma=\frac{E_{\text {supercell }}-\left(E_{\text {bulk }}^{\mathrm{Ta}}+E_{\text {bulk }}^{\mathrm{Cu}}\right)}{2 A}
$$

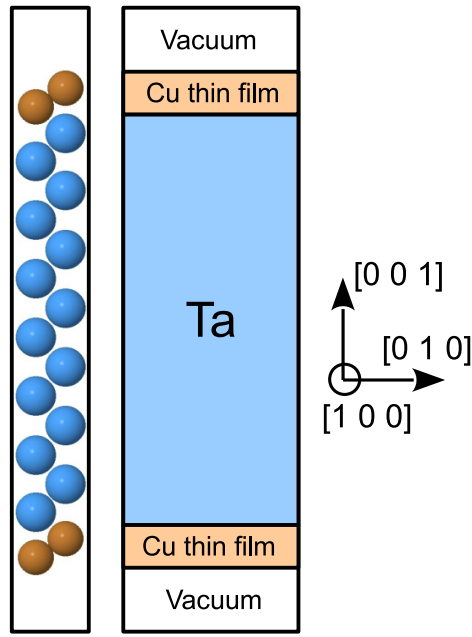

FIG. 3. Slab configuration to model the relaxation of a varying number of PM Cu layers.

where $E_{\text {supercell }}$ is the total energy of the slab calculation and $A$ is the surface area (considered twice for the two $\mathrm{Cu}-\mathrm{Ta}$ interfaces). The excess interface energy described in Eq. (1) is the sum of the film-substrate interface energy, $\gamma_{i}$, and the free surface energy of the thin film, $\gamma_{f}$, i.e., $\gamma=\gamma_{i}+\gamma_{f}$. The elastic strain energy contribution (estimated to $1 / 2 M \epsilon^{2} h$, with $M$ the biaxial in plane elastic modulus, $\epsilon$ the strain, and $h$ the thickness), which is implicitly contained in Eq. (1), increases with the number of layers of the film and quickly dominates the trend.

In order to evaluate the stability and growth mode of a film on a substrate, the energy difference $\delta$ between the free surface and the interface configurations needs to be considered. This is given by [31,32]:

$$
\delta=\gamma_{i}+\gamma_{f}-\gamma_{s}=\gamma-\gamma_{s},
$$

where $\gamma_{s}$ is the free surface energy of the substrate. For $\delta<0$ the film-substrate interface is thermodynamically stable and a complete coverage of the substrate by the thin film is expected. When this condition is satisfied, a layer-by-layer growth, known as the Frank-van-der-Merwe mode, is observed $[33,34]$. Starting from $\delta=0$, the Stranski-Krastanov growth is obtained, which corresponds to a $3 \mathrm{D}$ growth on top of the thin film. The excess interface energies calculated up to six $\mathrm{Cu}$ PM layers are reported in Table III.

The computed free surface energy, $\gamma_{f}$, of $\mathrm{Ta}(001)$ is 3.18 $\mathrm{J} / \mathrm{m}^{2}$, which is larger than the experimental value of $2.5 \mathrm{~J} / \mathrm{m}^{2}$ [35] but close to other theoretical values of $3.10 \mathrm{~J} / \mathrm{m}^{2}$ [36] and $3.14 \mathrm{~J} / \mathrm{m}^{2}$ [37]. This results in $\delta<0$ for up to five PM layers. As mentioned above, the increase in $\gamma$ found for increasing number of layers reflects the increase in strain energy.

TABLE III. Calculated excess interface energy $\gamma$ as a function of the number $N$ of PM Cu layers on the Ta(001) surface.

\begin{tabular}{lccccccc}
\hline \hline$N$ & 0 & 1 & 2 & 3 & 4 & 5 & 6 \\
\hline$\gamma\left(\mathrm{J} / \mathrm{m}^{2}\right)$ & 3.18 & 2.50 & 2.19 & 2.63 & 2.78 & 3.13 & 3.32 \\
\hline \hline
\end{tabular}




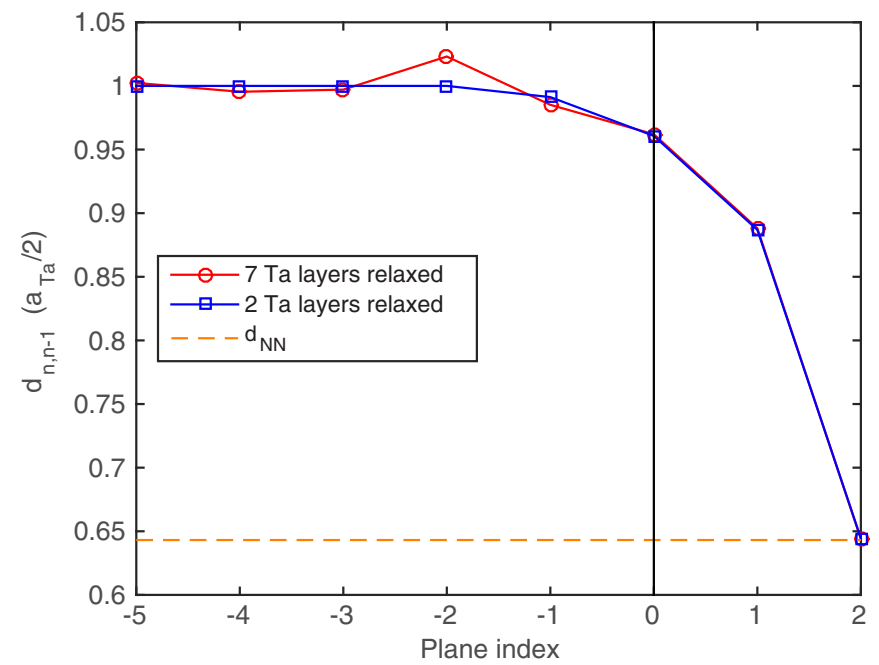

FIG. 4. Interplanar spacing versus plane index for two and seven relaxed Ta planes, normalized to the value in bulk tantalum $\left(a_{T a} / 2\right)$. The numerical values foseven relaxed planes are given in Table IV. The last tantalum plane is at index 0 ; indexes 1 and 2 correspond to copper planes. $d_{N N}$ is the value of $d_{1,2}$ that conserves the nearestneighbor distance of the bulk copper.

The configuration with two PM layers on top of the substrate has the lowest energy and is therefore the most stable configuration for a thin film of $\mathrm{Cu}$ on $\mathrm{Ta}(001)$ while one, three, four, and five $\mathrm{Cu}$ PM layers are metastable. The calculations indicate that a Frank-van-der-Merwe growth mode is expected up to five PM layers above which a Stranski-Krastanov mode is expected. This result is consistent with experimental data which reported the presence of one or two wetting layers [8]. For all the PM configurations, the high in-plane tensile strain induces a compensative high out-of-plane compressive strain among the $\mathrm{Cu}$ layers. The configuration with two PM layers is studied in more detail in the next section.

As a comparison, we computed the wetting behavior of $\mathrm{Cu}$ on $\mathrm{Ta}(110)$ and found that one PM layer is the most stable configuration thermodynamically, while two PM layers are metastable. The computed free surface energy of $\mathrm{Ta}(110)$ is $2.98 \mathrm{~J} / \mathrm{m}^{2}$ while the excess interface energy is $2.34 \mathrm{~J} / \mathrm{m}^{2}$ and $2.74 \mathrm{~J} / \mathrm{m}^{2}$, respectively. This result contradicts the findings of Ref. [11], where none of the PM configurations were predicted to be stable on the $\mathrm{Ta}(110)$ surface but is consistent with experimental observations $[7,10]$.

We also considered the case of $\mathrm{Cu}$ fcc layers on top of the $\mathrm{Ta}$ substrate or on top of the PM Cu layers. These configurations, requiring a significantly larger cell and larger computing time, did not converge to acceptable structures. For instance, the configuration with a single $\mathrm{Cu}$ fcc layer on top of the $\mathrm{Ta}$ substrate tends to form a PM layer. Other cases involving $\mathrm{PM} \mathrm{Cu}$ layers result in an out-of-plane modulation due to the periodic boundary conditions. It is therefore impossible to extract the excess interface energy of these configurations. We note that bcc copper is not uncommon at interfaces $[38,39]$.

The interplanar distances in the DFT configuration with two PM layers are shown in Fig. 4. The high in-plane misfit strain results in reduced interplanar distances along the (001) direction by $11 \%$ for the Ta-Cu interface and by $36 \%$ for the
$\mathrm{Cu}-\mathrm{Cu}$ bilayer, with respect to the interlayer spacing in bulk Ta $\left(a_{\mathrm{Ta}} / 2\right)$. Such a huge tetragonal distortion has been observed in thick films in the case of the Bain transformation [40]. Here it is not a relevant parameter of stability since we deal with only two atomic layers, each of them is at an interface. It is more relevant to consider the $\mathrm{Cu}-\mathrm{Cu}$ nearest-neighbor distance in the Cu bilayer. With an interplanar distance of $d_{1,2}=1.042 \AA$, the latter is

$$
d_{\mathrm{Cu}-\mathrm{Cu}}=\sqrt{2\left(\frac{a_{\mathrm{Ta}}}{2}\right)^{2}+d_{1,2}^{2}} \approx 2.513 \AA .
$$

By comparison, the nearest-neighbor distance in fcc copper $\left(a_{\mathrm{Cu}} / \sqrt{2}\right)$ is also $2.513 \AA$. (The real experimental value is $2.556 \AA$ at room temperature.) We see that, in the absence of a third layer, the body-centered tetragonal pseudomorphic stacking allows the copper atoms to keep an interatomic distance similar to that in the bulk fcc value, thus minimizing the elastic energy. A third PM layer would cause a large frustration either of the first-neighbor distance or of the secondnearest-neighbor distance, hence the increase of energy above two PM layers.

The interplanar distance at the interface is of $1.436 \AA$, resulting in a $\mathrm{Cu}-\mathrm{Ta}$ interatomic distance:

$$
d_{\mathrm{Cu}-\mathrm{Ta}}=\sqrt{2\left(\frac{a_{\mathrm{Ta}}}{2}\right)^{2}+d_{0,1}^{2}} \approx 2.701 \AA .
$$

The tantalum atoms are also affected by the presence of neighboring $\mathrm{Cu}$ atoms, and a strong relaxation of the first three tantalum interlayer distances at the interface is observed. The first two are compressed by $3.9 \%$ and $2.5 \%$ for $d_{-1,0}$ and $d_{-2,-1}$ respectively, while the third one is increased by $2.3 \%$. The strain below the first three planes nearly vanishes: The spacing between $\mathrm{Ta}_{-4}$ and $\mathrm{Ta}_{-5}, \mathrm{Ta}_{-5}$ and $\mathrm{Ta}_{-6}$, and $\mathrm{Ta}_{-6}$ and $\mathrm{Ta}_{-7}$ is indeed close to the bulk nominal value of $\mathrm{Ta}$ (respective variations of $-0.3 \%,-0.45 \%$, and $0.24 \%$ ).

\section{COMPARISON BETWEEN DFT CALCULATIONS AND SXRD DATA}

According to the DFT calculations, the thermodynamically most stable configuration is two PM copper layers on top of the $\mathrm{Ta}(001)$ surface. The agreement between DFT calculations and SXRD measurements was checked by computing the structure factors using the DFT configurations and comparing them with the experimental structure factors. These calculations were performed in the kinematical approximation of $\mathrm{x}$-ray diffraction using the software ROD [41] according to:

$$
F_{\text {tot }}(H K L) \propto\left|F_{b}(H K L)+F_{s}(H K L)\right|,
$$

where $H K L$ are the Miller indices. The bulk structure factor $F_{b}(H K L)$ is calculated for a perfect semi-infinite crystal of tantalum, and the surface structure factor $F_{s}(H K L)$ is calculated using all the atoms of the model, including the tantalum atoms belonging to the seven layers just below the copper layers:

$$
F_{S}(H K L)=\sum_{j} f_{j} e^{2 i \pi\left(H x_{j}+K y_{j}+L z_{j}\right)} e^{-\frac{B_{j}\left(H^{2}+K^{2}+L^{2}\right)}{4 a_{\mathrm{Ta}}^{2}}},
$$



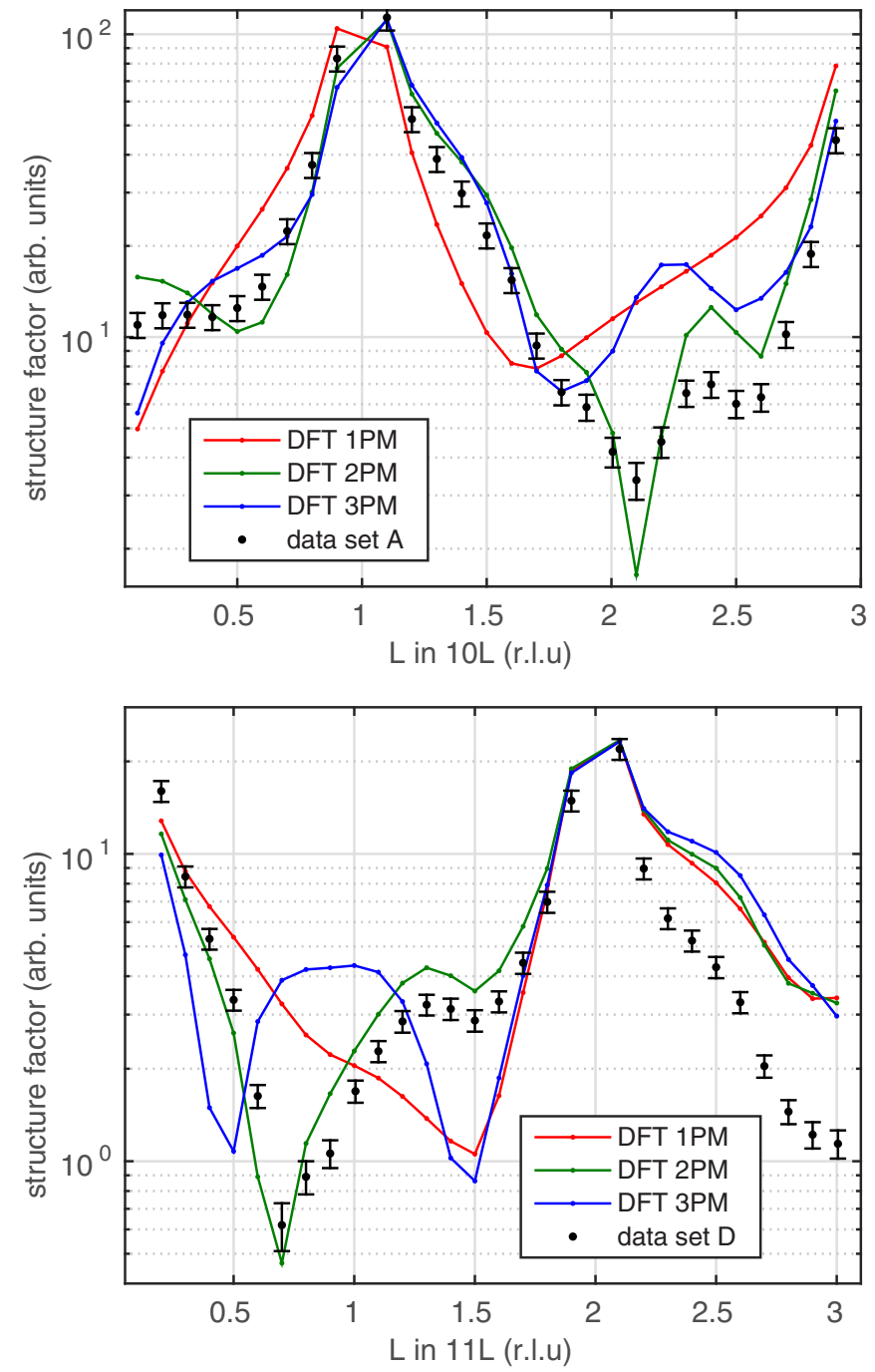

FIG. 5. $10 L$ (a) and $11 L$ (b) structure factors obtained using DFT configurations with a varying number of $\mathrm{PM} \mathrm{Cu}$ layers, compared with experimental data.

where $f_{j}$ is the atomic scattering factor of atom $j,(x y z)_{j}$ its position in fractional coordinates, and $B_{j}$ its isotropic Debye-Waller parameter. The scale factor in Eq. (5) includes a roughness factor calculated within the $\beta$ model [42].

Figure 5 presents several comparisons between CTRs measured from the cleanest Ta surfaces (data sets $\mathrm{A}$ and D), and the simulated CTRs for one, two, and three PM Cu layers. For both the $11 \mathrm{~L}$ and $10 \mathrm{~L}$ CTRs, the best agreement is found for two PM layers. We note that the structure factors are systematically overestimated at high $L$ values. Despite this discrepancy, the two-PM model reproduces well all the features of the experimental data, unlike the other models.

To clarify the importance of the Ta relaxation, the structure factors of four different systems were calculated and compared to the experimental data. In the first case, the relaxation of the $\mathrm{Cu}$ atoms was taken into account, whereas the atomic positions for Ta were equal to their nominal bulk values. In the second, third and fourth cases, the relaxation of $\mathrm{Cu}$ was taken in account and the relaxation of the Ta atoms was restricted respectively to two, three, and seven Ta planes. As shown in
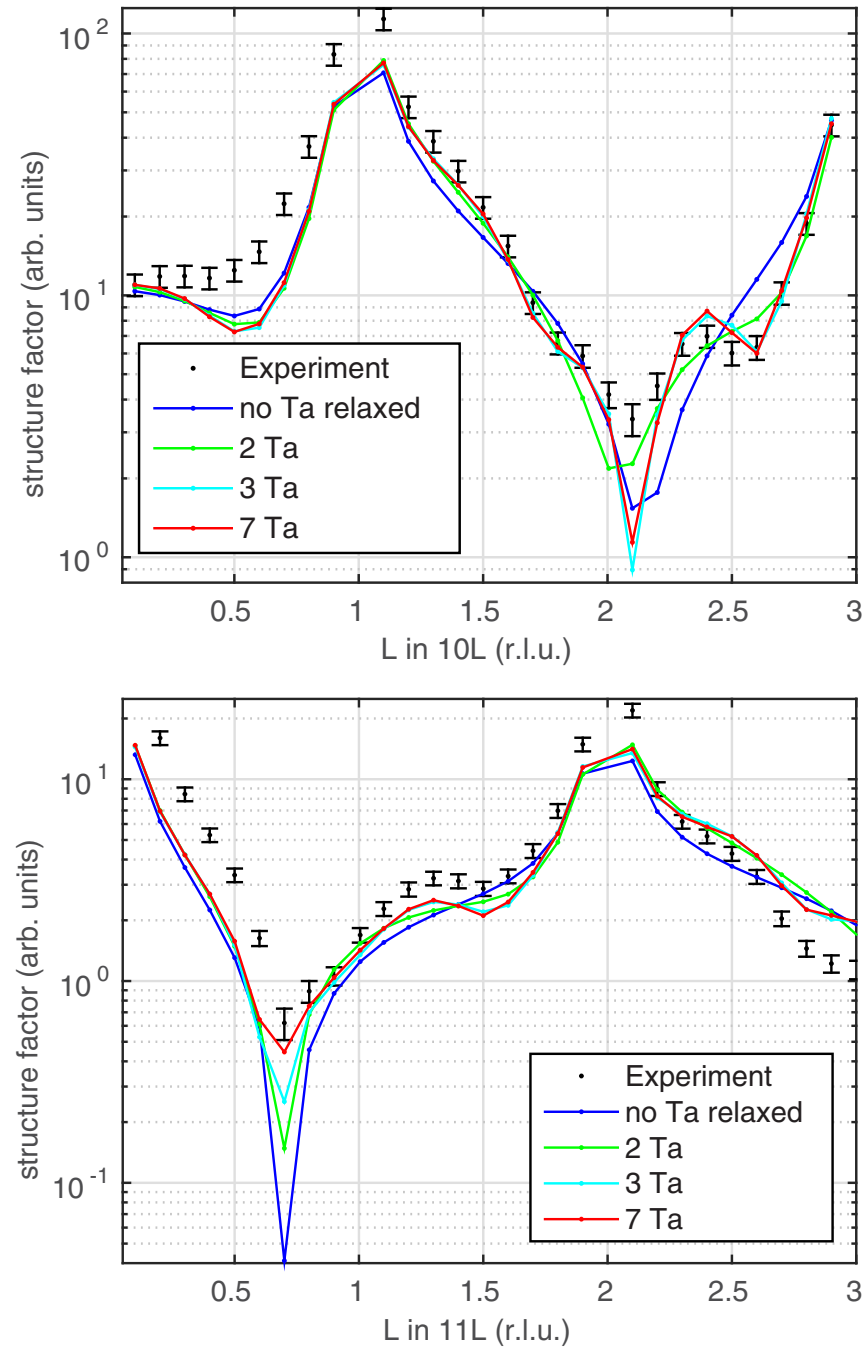

FIG. 6. Influence of the number of relaxed Ta planes in the DFT calculations on the calculated $10 L$ and $11 L$ structure factors.

Fig. 6, for up to three Ta planes, the number of Ta planes taken into account for relaxation has a very strong influence on the calculated structure factors. In all cases and independently of the number of relaxed Ta planes, the positions of the minima of amplitude are reproduced accurately for both CTR. However, the agreement between the calculated structure factors and the experimental data is rather poor for fewer than three relaxed Ta planes. For the $10 L \mathrm{CTR}$, the four configurations reproduce the dip of intensity around $L=2.1 \mathrm{rlu}$; however, the bump which is observed in the $2.2<L<2.6$ rlu region is reproduced accurately only when at least three Ta planes are relaxed. Similar conclusions can be drawn for the $11 L$ CTR where the configurations with zero and two relaxed Ta planes fail to reproduce the curve inflection in the $1<L<1.5$ rlu and $2.3<L<2.7$ rlu regions. The dip of amplitude for $L=0.7 \mathrm{rlu}$ is also strongly overestimated when the number of relaxed Ta planes is fewer than three. These remarks allow us to set an upper limit for the resolution obtained on the lattice relaxation: The relaxation of the third Ta layer, of $0.03 \AA$, is clearly seen. The simulated CTR for three and seven relaxed Ta planes exhibit a remarkable similarity. This suggests that 

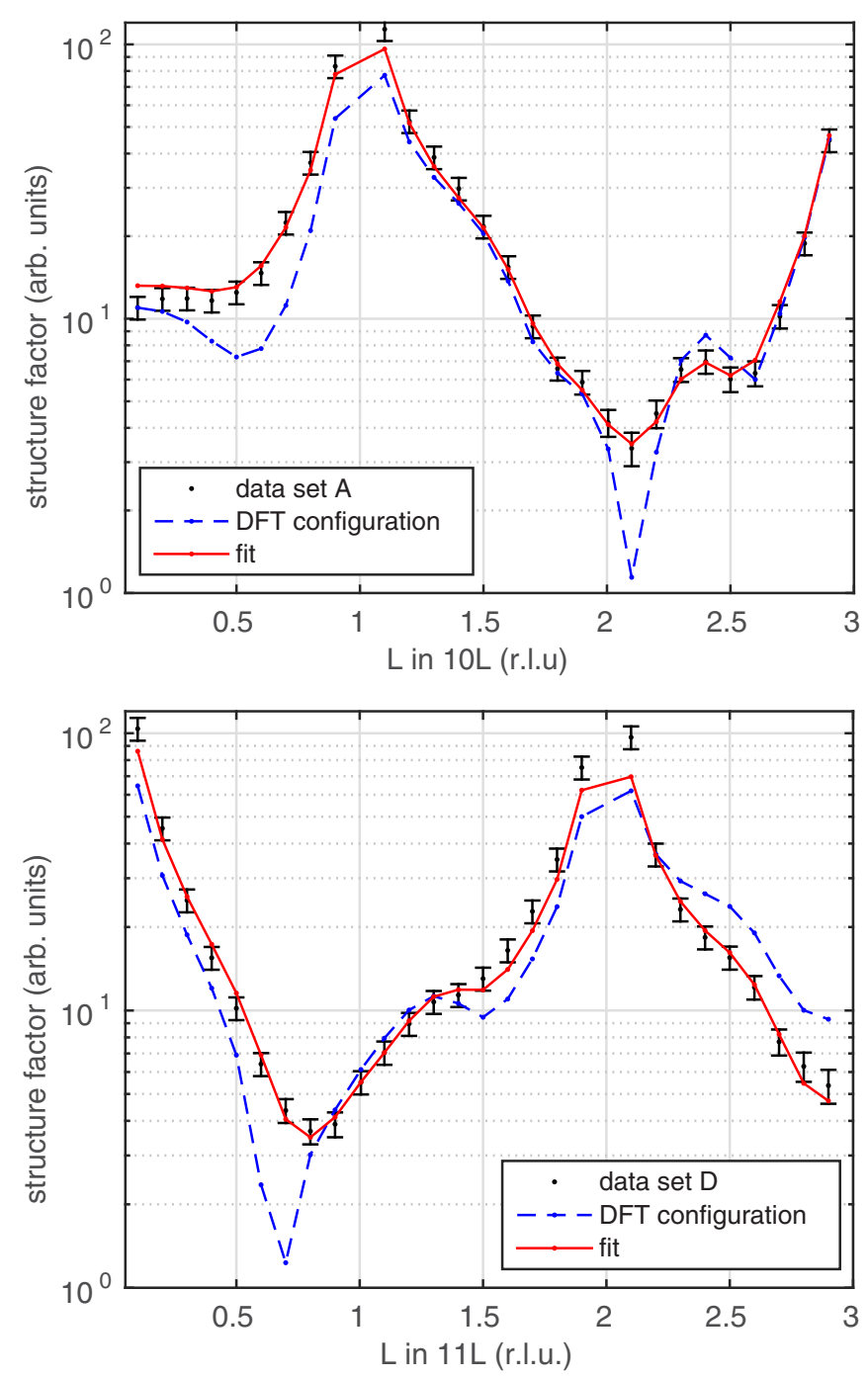

FIG. 7. $10 L$ and $11 L$ CTRs structure factors, before and after fitting.

most of the relaxation occurs within the upper three Ta planes and is consistent with the small value of displacements found for the Ta planes further away from the interface. The good agreement with the experimental data obtained by relaxing the atomic layers is strong evidence supporting the absence of nonpseudomorphic layers (fcc) on top of the pseudomorphic layers, because additional layers, even though invisible on the

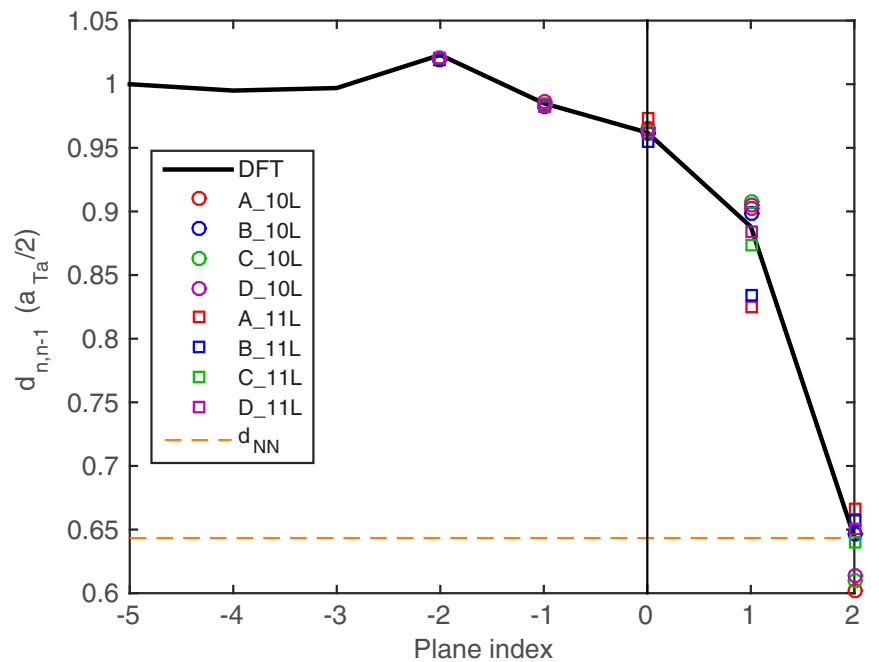

FIG. 8. Interlayer spacing resulting from the eight experimental curves. $d_{N N}$ is the value of $d_{1,2}$ that conserves the nearest-neighbor distance of the bulk copper. The values are given in unit of the interlayer spacing in the bulk tantalum.

CTRs originating from the Ta reciprocal lattice points, would modify the interplanar spacing.

Finally, the DFT structure of the fully relaxed interface with two PM layers was employed as the starting model to fit the experimental data by relaxing the following parameters: the interplanar spacings, the Debye-Waller factors, and the $\beta$ value of the roughness factor [42]. ROD also allows us to release the surface fraction of the coherent layer. Attempts to do so lead to values very close to 1 (full coverage) without significant improvements of the fits, and the results presented here were performed without releasing this parameter. Indeed, the total amount of deposited copper exceeded two PM and there is no reason to think that the surface coverage is not full. The eight experimental data sets (two CTRs for each of the four samples) were fitted independently. Figure 7 shows a comparison between two experimental curves and the calculations before and after fitting. The interlayer spacings resulting from the fits are given in Table IV, averaged by CTR over the four samples, and shown in Fig. 8. Alternative starting guesses were tried, such as using $a_{T a} / 2$ for the Ta-Ta interlayer distances, and the fits converged to the same values well within the error bars. Remarkably, the final fit parameters are very close to the DFT predictions. In particular, the SXRD

TABLE IV. Interlayer spacings: DFT values and refined fit parameters. Error bars for the CTR fits corresponds to the largest deviation to the mean in the four data sets (or in the $4 \times 2$ data sets for the average value). * Value not relaxed for the fits.

\begin{tabular}{|c|c|c|c|c|}
\hline Interlayer spacing $\left[/\left(a_{\mathrm{Ta}} / 2\right)\right]$ & DFT value & $10 L$ & $11 L$ & SXRD average \\
\hline$d_{-6,-5}(\mathrm{Ta}-\mathrm{Ta})$ & 1.000 & $1.000^{*}$ & $1.000^{*}$ & $1.000^{*}$ \\
\hline$d_{-5,-4}(\mathrm{Ta}-\mathrm{Ta})$ & 0.995 & $0.995^{*}$ & $0.995^{*}$ & $0.995^{*}$ \\
\hline$d_{-4,-3}(\mathrm{Ta}-\mathrm{Ta})$ & 0.997 & $0.997^{*}$ & $0.997^{*}$ & $0.997^{*}$ \\
\hline$d_{-3,-2}(\mathrm{Ta}-\mathrm{Ta})$ & 1.023 & $1.020 \pm 0.001$ & $1.020 \pm 0.001$ & $1.020 \pm 0.001$ \\
\hline$d_{-2,-1}(\mathrm{Ta}-\mathrm{Ta})$ & 0.985 & $0.985 \pm 0.002$ & $0.983 \pm 0.001$ & $0.984 \pm 0.003$ \\
\hline$d_{-1,0}(\mathrm{Ta}-\mathrm{Ta})$ & 0.962 & $0.964 \pm 0.002$ & $0.963 \pm 0.009$ & $0.964 \pm 0.009$ \\
\hline$d_{0,1}(\mathrm{Cu}-\mathrm{Ta})$ & 0.888 & $0.903 \pm 0.006$ & $0.85 \pm 0.03$ & $0.88 \pm 0.05$ \\
\hline$d_{1,2}(\mathrm{Cu}-\mathrm{Cu})$ & 0.644 & $0.62 \pm 0.03$ & $0.65 \pm 0.01$ & $0.64 \pm 0.03$ \\
\hline
\end{tabular}


data confirm the relaxation of the three upper atomic layers of tantalum. For each CTR, the dispersion of the values of the interlayer spacings is very small, suggesting the small influence of the surface contamination. There is actually more dispersion between the values provided by the two CTRs. This systematic error is also observed on the roughness parameter $\beta$, which is found of the order of $1 \%$ for the $10 \mathrm{~L}$ CTR and of the order of $10 \%$ for the $11 \mathrm{~L} \mathrm{CTR}$. In addition to the interlayer spacings, the isotropic Debye-Waller factors of the four upper layers (the two $\mathrm{Cu}$ layers and two Ta layers) are relaxed for the fits. The fits yield isotropic Debye parameters of 3.8, 4.7, 5.3, and $11.1 \AA^{2}$ from bottom to top, with dispersions close to $100 \%$. These large values are needed to reproduce the fall of the experimental data at large $L$. We infer that they point to a large static disorder. The surface contamination and its increase with time have probably a major role in determining such high values.

\section{CONCLUSIONS}

This study of the $\mathrm{Cu}-\mathrm{Ta}(001)$ interface, combining SXRD measurements and DFT calculations in excellent mutual agreement, demonstrates the presence of two pseudomorphic layers of copper wetting on the Ta(001) surface after dewetting of excess copper. This result is robust for a small contamination of the surface by carbon and oxygen. The measurements performed with the lowest contamination were used to fit the surface structure and to determine the interplanar distances. The two copper PM layers are in body-centered tetragonal stacking and have interplanar spacing of $d_{1,2}=1.05 \AA \pm$ $0.05 \AA$, which maintains the same nearest-neighbor distance as in the bulk (fcc). This configuration, which minimizes the elastic energy in the $\mathrm{Cu}-\mathrm{Cu}$ bonds, is possible only in the absence of a third layer of copper. This feature is possibly one reason that explains the anomalously fast diffusion of $\mathrm{Cu}$ atoms during solid-state dewetting on $\mathrm{Ta}(001)$. Indeed, the dewetting occurs not directly on the $\mathrm{Ta}(001)$ surface but on top of two PM layers.

\section{ACKNOWLEDGMENTS}

Beamtime is acknowledged at the French CRG BM32 beamline of the ESRF. Calculations were performed using computer resources from GENCI under CINES Grant No. C2016097211 and the Froggy platform of the CIMENT infrastructure, which is supported by the Rhône-Alpes region (GRANT CPER07 13 CIRA). This work was supported by the French ANR Project No. ANR-11-BS10-0014 (MECANIX).
[1] K. Holloway, P. M. Fryer, C. Cabral Jr, J. Harper, P. Bailey, and K. Kelleher, J. Appl. Phys. 71, 5433 (1992).

[2] T. Laurila, K. Zeng, J. K. Kivilahti, J. Molarius, and I. Suni, J. Appl. Phys. 88, 3377 (2000).

[3] H. Kim, T. Koseki, T. Ohba, T. Ohta, Y. Kojima, H. Sato, and Y. Shimogaki, J. Electrochem. Soc. 152, G594 (2005).

[4] C. M. Müller and R. Spolenak, Thin Solid Films 598, 276 (2016).

[5] M. F. Singleton, J. L. Murray, and E. Nash, Binary Alloy Phase Diagrams, edited by T. B. Massalski, 2nd edn. (ASM International, Materials Park, 1990), p. 181.

[6] J. A. Rodriguez and D. W. Goodman, J. Phys. Chem. 95, 4196 (1991).

[7] L. Chen, N. Magtoto, B. Ekstrom, and J. Kelber, Thin Solid Films 376, 115 (2000).

[8] V. Venugopal and B. Thijsse, Thin Solid Films 517, 5482 (2009).

[9] F. Fillot, Z. Tőkei, and G. Beyer, Surf. Sci. 601, 986 (2007).

[10] W. K. Kuhn, R. A. Campbell, and D. W. Goodman, J. Phys. Chem. 97, 446 (1993).

[11] A. Hashibon, C. Elsässer, Y. Mishin, and P. Gumbsch, Phys. Rev. B 76, 245434 (2007).

[12] M. F. Francis, M. N. Neurock, X. W. Zhou, J. J. Quan, H. N. G. Wadley, and E. B. Webb, J. Appl. Phys. 104, 034310 (2008).

[13] G. Beutier, M. Verdier, G. Parry, B. Gilles, S. Labat, M.-I. Richard, T. Cornelius, P.-F. Lory, S. V. Hoang, F. Livet et al., Thin Solid Films 530, 120 (2013).

[14] H. R. Gong and B. X. Liu, Appl. Phys. Lett. 83 (2003).

[15] I. Lazić, P. Klaver, and B. Thijsse, Phys. Rev. B 81, 045410 (2010).

[16] A. Hashibon, A. Y. Lozovoi, Y. Mishin, C. Elsässer, and P. Gumbsch, Phys. Rev. B 77, 094131 (2008).

[17] I. K. Robinson and D. J. Tweet, Rep. Prog. Phys. 55, 599 (1992).

[18] S. R. Andrews and R. A. Cowley, J. Phys. C 18, 6427 (1985).
[19] M. Ball, C. Lucas, N. Markovic, V. Stamenkovic, and P. Ross, Surf. Sci. 518, 201 (2002).

[20] R. Baudoing-Savois, M. D. Santis, M. Saint-Lager, P. Dolle, O. Geaymond, P. Taunier, P. Jeantet, J. Roux, G. Renaud, A. Barbier, O. Robach, O. Ulrich, A. Mougin, and G. Bérard, Nucl. Instrum. Methods Phys. Res. B 149, 213 (1999).

[21] J. M. Bloch, J. Appl. Crystallogr. 18, 33 (1985).

[22] R. Musket, W. McLean, C. A. Colmenares, D. Makowiecki, and W. Siekhaus, Appl. Surf. Sci. 10, 143 (1982).

[23] H. Yamazaki, K. Sakamoto, A. Fujii, and T. Kamisawa, Surf. Sci. 563, 41 (2004).

[24] C. Ponchut, J. Rigal, J. Clément, E. Papillon, A. Homs, and S. Petitdemange, J. Instrum. 6, C01069 (2011).

[25] E. Vlieg, J. Appl. Crystallogr. 30, 532 (1997).

[26] E. Vlieg, From beam time to structure factors, including a concise guide to ANA and AVE (2012), http://www. esrf.eu/computing/scientific/joint_projects/ANA-ROD/binaries /anamanual.pdf.

[27] K.-W. Kwon, H.-J. Lee, and R. Sinclair, Appl. Phys. Lett. 75 (1999).

[28] N. Troullier and J. L. Martins, Phys. Rev. B 43, 1993 (1991).

[29] P. Giannozzi, S. Baroni, N. Bonini, M. Calandra, R. Car, C. Cavazzoni, D. Ceresoli, G. L. Chiarotti, M. Cococcioni, I. Dabo et al., J. Phys.: Condens. Matter 21, 395502 (2009).

[30] H. J. Monkhorst and J. D. Pack, Phys. Rev. B 13, 5188 (1976).

[31] L. B. Freund and S. Suresh, Thin Film Materials: Stress, Defect Formation and Surface Evolution (Cambridge University Press, Cambridge, 2004).

[32] M. Wuttig and X. Liu, Ultrathin Metal Films: Magnetic and Structural Properties (Springer, Berlin Heidelberg, 2004), pp. 125-207.

[33] F. C. Frank and J. H. van der Merwe, Proc. R. Soc. London A (1949). 
[34] J. H. Van Der Merwe, J. Appl. Phys. 34, 123 (1963).

[35] W. Tyson and W. Miller, Surf. Sci. 62, 267 (1977).

[36] L. Vitos, A. Ruban, H. L. Skriver, and J. Kollar, Surf. Sci. 411, 186 (1998).

[37] F. Aqra and A. Ayyad, Appl. Surf. Sci. 257, 6372 (2011).

[38] C. Schmidt, F. Ernst, M. W. Finnis, and V. Vitek, Phys. Rev. Lett. 75, 2160 (1995).
[39] H. Kung, Y.-C. Lu, A. J. Griffin, M. Nastasi, T. E. Mitchell, and J. D. Embury, Appl. Phys. Lett. 71, 2103 (1997).

[40] J. Buschbeck, I. Opahle, M. Richter, U. K. Rößler, P. Klaer, M. Kallmayer, H. J. Elmers, G. Jakob, L. Schultz, and S. Fähler, Phys. Rev. Lett. 103, 216101 (2009).

[41] E. Vlieg, J. Appl. Crystallogr. 33, 401 (2000).

[42] I. K. Robinson, Phys. Rev. B 33, 3830 (1986). 